\title{
Image-Guided Breast Interventions: Biopsy and Beyond
}

\author{
Ekta Dhamija ${ }^{1, \odot ~ R a s h m i ~ S i n g h ~}{ }^{1}$ Seema Mishra² \\ ${ }^{1}$ Department of Radiodiagnosis, Dr. B.R.A. Institute Rotary Cancer \\ Hospital, All India Institute of Medical Sciences, New Delhi, India \\ ${ }^{2}$ Department of Oncoanaesthesia \& Palliative Medicine, Dr. B.R.A. \\ Institute Rotary Cancer Hospital, All India Institute of Medical \\ Sciences, New Delhi, India
}

\author{
Smriti Hari ${ }^{1}$
}

\begin{abstract}
Address for correspondence Dr. Ekta Dhamija, MD, Department of Radiodiagnosis, Dr. B.R.A. Institute Rotary Cancer Hospital, All India Institute of Medical Sciences, Room number 137, First Floor, New Delhi 110029, India (e-mail: drektadhamija.aiims@gmail.com).
\end{abstract}

\begin{abstract}
Keywords

- breast cancer

- breast biopsy

- core needle biopsy

- vacuum needle biopsy

- tumor localization

- breast Interventions

Breast interventions primarily comprise of biopsy of the suspicious breast lesions to obtain accurate pathological diagnosis. Generally, image-guided breast biopsy is required for nonpalpable lesions, however, even in palpable lesions, image-guided biopsy should be performed as it improves the accuracy of diagnosis. Image-guided breast interventions have progressed well beyond biopsy, making the radiologist an important part of the multidisciplinary management of breast cancer. Preoperative localization of nonpalpable abnormalities guides optimal surgical excision to obtain negative margins without sacrificing the normal tissue. Ablative procedures for breast cancer treatment such as radiofrequency ablation (RFA) and high-intensity focused ultrasound ablation can sometimes replace surgery in older patients with comorbidities. This article enumerates and describes the expanding spectrum of image-guided interventions performed by breast radiologist.
\end{abstract}

\section{Introduction}

Breast cancer is the most common cancer affecting women in India. It accounts for $26.3 \%$ of all new cancer diagnoses and $23.5 \%$ of all cancer deaths among women in India. ${ }^{1,2}$ Multidisciplinary approach, personalized treatment, breast conservation treatment have increased the scope for breast radiologist not only in diagnosis but also in its treatment

Breast biopsy of suspicious abnormalities is the most common image-guided breast intervention performed as image guidance enables accurate tissue sampling and reduces need of repeat biopsies as compared with blind biopsies. ${ }^{3}$ Only after confirmation on histopathology, further treatment is planned, based on the hormonal receptor status of tumor. Increasing use of neoadjuvant chemotherapy (NACT) in larger tumors before surgery makes breast conservative surgery (BCS) possible in patients showing good response. ${ }^{4}$

published online

July 28, 2021
DOI https://doi.org/

$10.1055 / \mathrm{s}-0041-1734223$ ISSN 0971-3026
With newer, improved chemotherapeutic agents, nearly 20 to $25 \%$ patients may show complete clinical and radiological response to NACT. However, it also mandates localization of tumor site before or during chemotherapy before the tumor vanishes completely, using tumor marker clips. These marker clips serve as localization site for subsequent image-guided hookwire placement before surgery.

This article highlights expanding role of radiologist beyond biopsy in the field of breast radiology ( - Fig. 1 ).

\section{Breast Biopsy}

The techniques and instrumentation of image-guided breast biopsy are different from those required for biopsies of other body parts. Breast lesions classified as BIRADS 4 (suspicious) or BIRADS 5 (highly suspicious) on imaging are primary indications for breast biopsy. Larger palpable lesions undergo blind percutaneous biopsy and nonpalpable small, deep

(C) 2021. Indian Radiological Association.

This is an open access article published by Thieme under the terms of the Creative Commons Attribution-NonDerivative-NonCommercial-License, permitting copying and reproduction so long as the original work is given appropriate credit. Contents may not be used for commercial purposes, or adapted, remixed, transformed or built upon. (https://creativecommons.org/licenses/by-nc-nd/4.0/).

Thieme Medical and Scientific Publishers Private Ltd. A-12, Second Floor, Sector -2, NOIDA -201301, India 


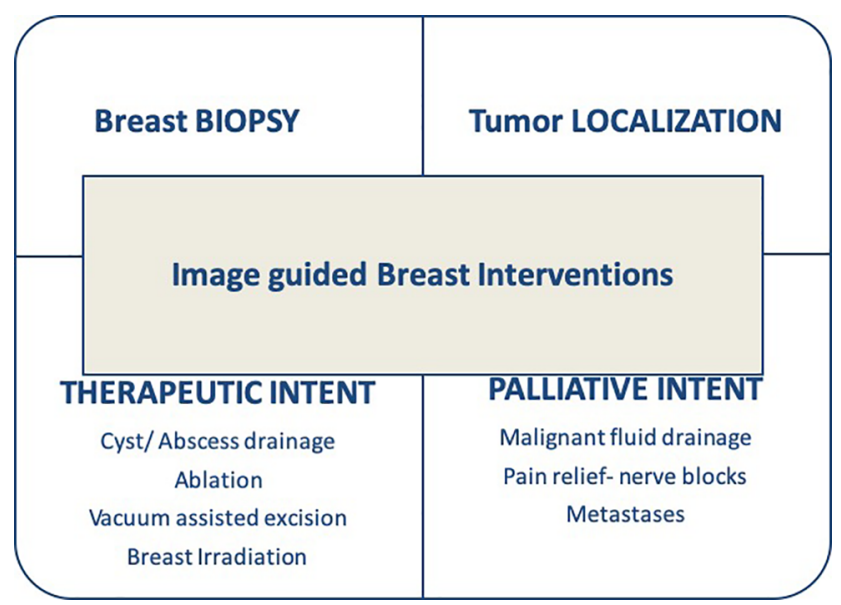

Fig. 1 Flow diagram illustrating the expanding scope of image-guided breast interventions.

seated or vaguely palpable masses would need image-guided biopsy. Blind biopsy, however, is associated with high false negative and repeat biopsy rates emphasizing the need of image-guided biopsies even in the setting of palpable lesions due to sampling error. ${ }^{3}$ Image-guided breast biopsy can be performed under ultrasound, mammography (stereotactic), or MR guidance, depending on the visibility of the lesion and operator preference. Ultrasound is the most commonly used modality, whenever the breast lesion can be unequivocally demonstrated on ultrasound (US). Microcalcifications and masses seen on mammogram will undergo stereotactic biopsy. Similarly, MR-guided biopsy can be performed for lesions visible only on MRI.

\section{FNAC versus Core Biopsy}

FNAC performed with 20 to 22 -gauge needles is a simple, less expensive, and less traumatic technique. However, FNAC has high insufficiency and false negative rates. ${ }^{5}$ It also requires the on-site availability of an experienced breast cytologist. FNAC cannot differentiate in-situ from invasive cancer and immuno-histochemical analysis for hormonal receptor status is difficult on the fine-needle aspirate. Percutaneous core needle biopsy ( $\mathrm{CNB}$ ) of the breast is more accurate than FNAC and this has become the standard of care for biopsy of breast lesions. However, it is more traumatic and requires higher operator skill when compared with FNAC. Breast CNB is commonly performed using spring loaded automated 14-gauge core biopsy needles, commonly referred to as biopsy guns. Small caliber (16 or 18 gauge) biopsy needles used for other parts of the body are not suitable for breast biopsy. Typically, a minimum of four to five cores should be obtained during breast CNB. ${ }^{6}$

\section{Vacuum-Assisted Biopsy}

Vacuum-assisted biopsy (VAB) of breast is a modified and improved technique of large core breast biopsy that was introduced in late 1990s. ${ }^{7}$ In this device, the probe (needle) consists of a motor driven outer cannula with the sample notch and an inner cutting cannula. Strong vacuum sucks and holds the targeted tissue in the sample notch and transports the harvested cores outside into a collection chamber. VAB devices allow directional sampling from different parts of the lesion obtaining around 12 to 20 cores while rotating the needle through 360 degrees. Some of the currently available VAB devices include Mammotome (Ethicon Endosurgery, Ohio, United States), Eviva (Hologic, Beford, United States), and Senorex (Bard, Tempe, United States). VAB uses thick calibers ranging from 11 to 7 gauge depending on the vendor/apparatus. Single time insertion with faster acquisition of multiple large tissue cores in contiguous manner is the major advantage with VAB. In addition, it allows irrigation of the biopsy cavity at the same time with saline and local anesthetic agent. Ability to continuously irrigate the biopsy site with saline and apply strong suction reduces the chances of hematoma formation. Since VAB can remove large amount of tissue, smaller lesions may get removed completely during the biopsy. This necessitates placement of marker clip at the biopsy site to guide surgery in case the histopathology result is positive for cancer, which can be done in same sitting and through same notch/aperture without removing the needle. VAB has greater accuracy than CNB in terms of the frequency of histological underestimation, false negatives, and need for rebiopsy. With negative predictive value reaching up to $99.9 \%,{ }^{8,9} \mathrm{VAB}$ is considered to be as good as excisional surgical biopsy..$^{10} \mathrm{VAB}$, however, is not widely available, is more expensive than CNB using biopsy gun and has a definite learning curve. At our institute, VAB is performed for all stereotactic and MRI-guided biopsies whereas in case of ultrasonography (USG) guidance, it is often used only if the pathological analysis is incomplete or inconclusive (-Fig. 2).

\section{Digital Breast Tomosynthesis-Guided Biopsy}

DBT provides pseudo-three-dimensional images of the breast leading to optimum visualization of subtle findings such as

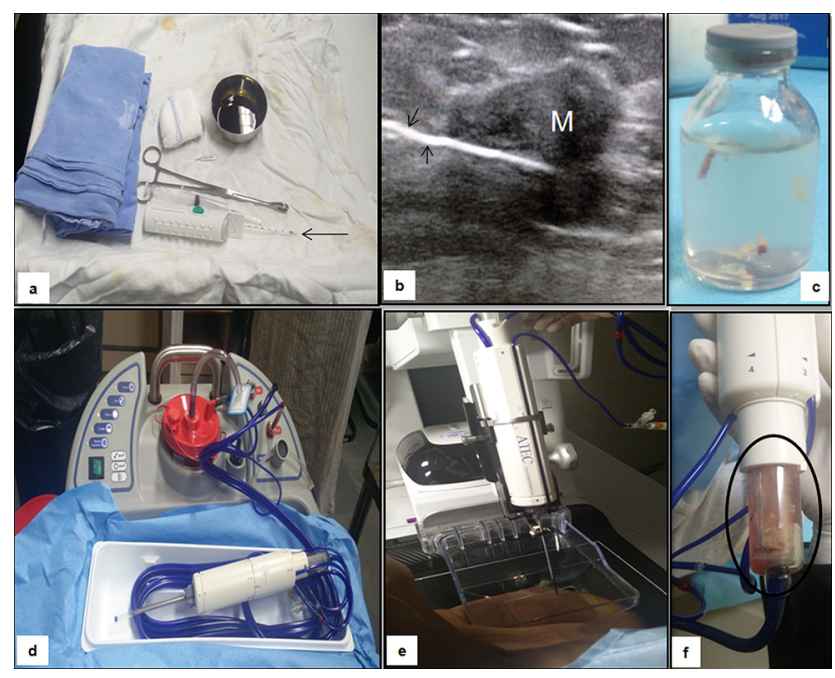

Fig. 2 Breast biopsy. Panel $(\mathbf{a}-\mathbf{c})$ demonstrates prepared sterile tray with 14-gauge core biopsy needle (arrow in a) with sterile drapes and other accessory items needed. The cores obtained under USG guidance (arrows in b) have settled or are semi-floating in the container (c). On the other hand, apparatus for vacuum-assisted biopsy (d-f) is complex (d) and the cores get collected in sample notch using vacuum (f) which is then shifted to the formalin bottle. 
asymmetries, masses, and architectural distortion. ${ }^{11,12}$ Before the advent of DBT-guided biopsy, architectural distortion seen only at DBT required surgical excision. ${ }^{13}$

The procedure involves compression of breast followed by DBT exposure to identify the target lesion and its marking using three-time exposure-first for localization, second for prefire, and last post sampling to confirm clip deployment. The study by Bahl et a ${ }^{14}$ concluded that overall technical and sampling success was more for DBT-guided VAB as compared with stereotactic VAB with additional advantage of less radiation exposure and time consumption with the former.

\section{Tumor Localization Techniques}

BCS is increasingly being performed for patients with small tumor to breast volume ratio allowing locoregional disease removal with clear margins and good cosmesis. NACT reduces the tumor burden in locally advanced breast cancer rendering BCS possible rather than mastectomy. ${ }^{15}$ In many of these patients, NACT achieves complete resolution of clinically palpable mass and radiological abnormality before surgery. There is a need to accurately localize the tumor site at the time of surgical excision to establish complete pathological response. ${ }^{16,17}$ Various innovative techniques like skin tattooing, imaging-guided radio-opaque tumor marker placement ranging from custom made clips using angiographic wire, commercially available marker clips to radioactive seed (I-125), Magseeds, and radiofrequency identification have been described. . $18,18,19,20,21,22,23$ Placement of tumor marker clips improves the locoregional disease control irrespective of the tumor stage with reduced incidence of local recurrence as compared with those who undergo surgery without marker placement. ${ }^{24}$

\section{Preoperative Hook Wire Localization}

This procedure was performed routinely for surgical excision biopsy of nonpalpable lesions. However, after advent of stereotactic biopsy, it is mainly used as a preoperative localization procedure for therapeutic excision of already diagnosed but nonpalpable breast cancer (small screening detected cancers or cancers rendered nonpalpable by NACT). Placement of two or more hookwires in the same breast may be required for multiple lesions to mark the extent of a large lesion and for segmental microcalcifications before breast conservation surgery. ${ }^{25}$ This is known as hookwire bracketing as shown in - Fig. $3 .^{26}$ Needle localization prior to breast conservation surgery helps to precisely resect the cancer with clear margins. Various needle-hookwire combinations are used for this procedure and Kopan's needle-hookwire assembly is most widely used in our setup. It consists of a 20-gauge needle and a thin flexible wire that can be introduced through it. The distal end of the wire folds back forming a sharp angle or has retractable curved J tip. Needle localization is usually performed under mammographic or US guidance on the same day of surgery. MR compatible needle hookwire assemblies are also available to localize the lesions seen only on MRI.

The surgeon dissects the breast using hookwire as a guide. Approximate part of the breast tissue around the hook and

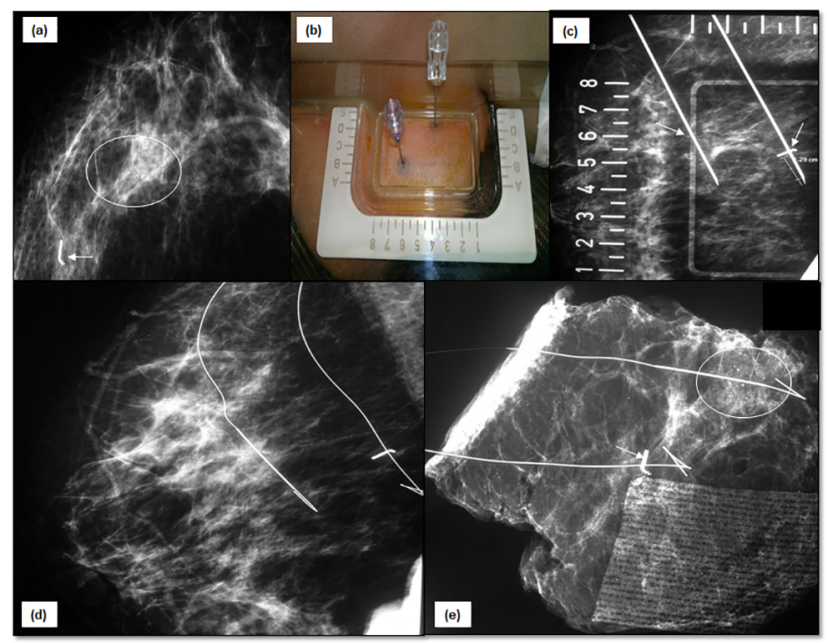

Fig. 3 Hookwire bracketing: (a) Mammogram of right breast shows tumor marker clip (arrow) at the site of mass which has resolved post chemotherapy. There is another cluster of suspicious microcalcification in same quadrant (circle). (b) Both the clips and grouped microcalcifications are targeted using alphanumeric grid and two hookwires have been placed (arrows in c). This procedure is known as "hookwire bracketing." The position of wires is confirmed on MLO view (d). Subsequently performed specimen mammogram (e) demonstrates and ensures removal of marker clip (arrow), microcalcifications (circle) and hookwires.

the thickened wire segment is resected and sent to the mammography room for specimen radiography. The specimen radiography should be done on the mammography unit with minimal exposure factors. It should confirm that the target lesion, along with good margins, has been removed in the resected specimen. Surgeon waits in operation theater for information regarding successful removal of the lesion, clip, and hookwire before closing the breast ( $\boldsymbol{- \text { Fig. }} \mathbf{4}$ ).

The procedure is safe with few minor complications like pain, vasovagal reaction, and bleeding. Rare complications like hooking of wire to pectoralis muscle and pneumothorax have been reported in few patients. ${ }^{27}$ Inadvertent pulling out of the hookwire after successful placement is a concern in fatty breasts due to arm movement by patient or improper handling during surgery. ${ }^{28} \mathrm{~A}$ failure rate of 0 to $18 \%$ has been reported in literature that is seen more often when mammography is used as the imaging modality for wire placement. ${ }^{27}$ Rates of negative margins after wire-localized BCS have been reported to range between 70.8 and $87.4 \%{ }^{29}$

Newer localization techniques include radioactive seed localization which can overcome the difficulties associated with wire localization such as displacement of wire, kinking/fracturing of wire, patients discomfort, difficulty in planning surgical incision, scheduling radiological and surgical procedure on the same day. Significant difference in negative margins rates has been observed between two techniques $-73 \%$ in radioactive seed localization versus $54 \%$ in wire localization. ${ }^{30}$

The seeds can be inserted under USG or mammographic guidance within the lesion 5 to 7 days prior to surgery and correct positioning is confirmed using mammography. The radioactive seed $\left(\mathrm{I}^{125}\right)$ is localized intraoperatively with a 

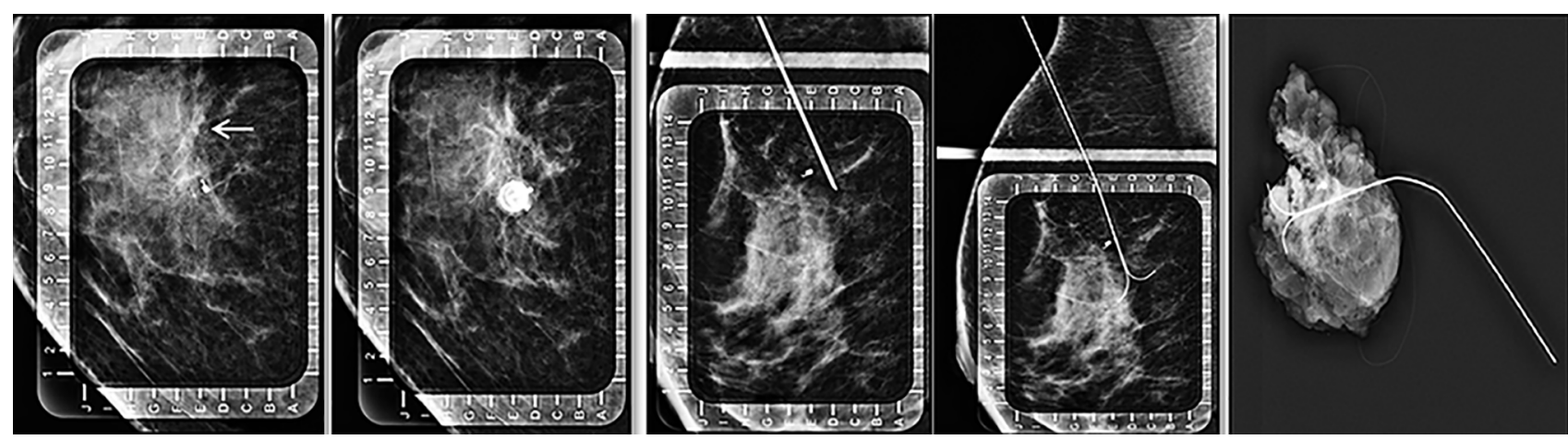

Fig. 4 Tumor localization using hook wire (left to right): the tumor site is identified on mammogram (white arrow) and targeted based on alphanumeric compression plate followed by insertion and deployment of the hookwire. Radiograph of surgical specimen is then obtained which confirmed removal of clip as well as hookwire in toto.

hand-held gamma probe. Most of the seeds are used with radiation exposure within safe limits with involvement of radiation safety officer in proper handling, use and disposal of radioactive seed. ${ }^{31}$ Limitation of this technique is that once the seed is deployed within the breast, it cannot be adjusted. If necessary, second seed placement might be required for correct localization. ${ }^{32}$

On the other hand, Megaseed, which is composed of paramagnetic steel and iron oxide, is not associated with radiation exposure. The insertion can be done 2 to 30 days prior to the surgery and a dedicated Sentimag detector detects the seed during the surgery. Being costlier is its major limitation. ${ }^{33}$

Intraoperative ultrasound (IOUS) is an underutilized technique that can be easily performed preoperatively to guide surgery. It reduces the rate of re-excision in cases of BCS by ensuring enough margins around the mass during removal. USG performed during surgery enables real time visualization of mass and its margins improving the accuracy of tissue removal. It also reduces the volume of normal breast tissue removed by virtue of assessing the specimen in the operation theater itself. Studies comparing IOUS and wire-guided localization in achieving negative resection margins showed that IOUS is at least equal or more successful than latter. ${ }^{34}$ $\mathrm{Hu}$ et al in year 2020 showed positive margin rate in IOUS was $4.6 \%$ as compared with $19.4 \%$ in the wire localization group. ${ }^{35}$ Adequate excision, negative margins with small excision volume is possible with IOUS as it measures distance from the tumor border to the resection margin and achieves better cosmesis as well. ${ }^{36,37}$

\section{Interventions with Therapeutic Intent Cyst Aspiration and Abscess Drainage}

Breast cysts are often seen while evaluating patients with nonspecific breast complaints or cyclical breast pain. Simple cysts are anechoic with only fluid within and thin imperceptible walls-usually asymptomatic until large in size. Complicated cyst refers to cyst with secondary infection or hemorrhage identified as dense internal echoes within a cyst with or without moving fluid or fluid-debris levels. These two types of cysts need intervention only if they are symptomatic and interfering with quality of life of the patient. ${ }^{38}$ On the contrary, complex cyst means cyst with thick internal septations or mural nodule showing vascularity that raises suspicion for associated malignancy and thus these complex cysts should be biopsied.

When indicated, cyst aspiration is performed under aseptic precautions under real time USG guidance and fluid should be sent for cytology. Most of the time single aspiration is sufficient; however, in many cases, re-accumulation of fluid occurs. Recurrence rate following simple cyst aspiration is variable, ranging from 20 to $80 \%{ }^{39,40}$ Sclerotherapy is emerging technique to overcome the drawbacks of recurrence associated with aspiration. In this procedure, most of the cyst volume is aspirated and minimal safe residual volume is left such that needle tip remains within the residual cyst cavity. Without removing needle, $99 \%$ ethanol with volume equivalent to the $90 \%$ of the aspirated fluid is injected and it is re-aspirated completely after 10 minutes. Burning sensation and pain are the common complications of this procedure. It has been reported that percutaneous ethanol sclerotherapy in simple breast cyst is an effective treatment with $100 \%$ clinical success rate on follow-up. ${ }^{41}$ Other techniques such as air instillation and administration of sclerosing solution (sclerovein) following aspiration of cysts have been described with 16 and $2 \%$ recurrence rate. ${ }^{39,40}$ None of these sclerotherapy techniques are practiced at authors' institute.

Infected simple cysts or de novo breast abscesses are seen in young lactating women, also in nonlactating smoker and elderly women. These can be categorized as puerperal (within 12 weeks of delivery) and nonpuerperal (during nonbreastfeeding period) abscess. Previously, surgical incision and drainage were the recommended treatments for breast abscesses. Since this is associated with the development of cutaneous fistulas in 5 to $12 \%$ of patients and recurrences in 10 to $38 \%$ of cases $^{42}$, US-guided intervention is now a preferred approach for the management due to its minimal invasive nature, easy accessibility, and shorter procedure time. It is associated with no significant scarring, needs no admission and breast feeding needs not be discontinued. Aspiration is followed by saline lavage of cavity till clear saline is aspirated. Usually, repeated aspirations and percutaneous catheter drainage are required for complete resolutions in abscesses more than $3 \mathrm{~cm}$ in size. ${ }^{43}$ Percutaneous catheter drainage is not commonly done and is reserved for failed repeated 
aspirations (at least five attempts of aspiration). 6F- or 8 F-sized catheters are used for drainage. ${ }^{44}$

\section{Vacuum-Assisted Excision of Benign Tumors}

Presence of benign lesions and lumps elevates patient's anxiety and may cause cosmetic disfigurement. Surgical excision is the preferred treatment option in such scenario. However, it is associated with incision site scarring as well as the need for localization in nonpalpable lesions. USG-guided VAB can achieve complete excision of lesions less than $2 \mathrm{~cm}$ in maximum diameter and the lesions with predominant cystic component. ${ }^{45}$ Completeness of procedure can be assessed with nonvisualization of lesion on US and absence of lesion in extracted specimen. ${ }^{46}$ Clip is usually inserted following procedure completion especially in patients with solid cystic masses. Hematoma, pain, and ecchymosis are common complications associated with it. ${ }^{47}$ For lesions less than $2 \mathrm{~cm}$, 95 to $100 \%$ of complete resection rate has been reported. ${ }^{46,48,49}$

\section{Radiofrequency Ablation}

There has been a paradigm shift in the treatment of early breast cancer with an attempt of using minimally invasive technique achieving complete tumor removal preserving significant functional breast volume. Several studies have confirmed the feasibility of radiofrequency ablation (RFA) followed by surgery as well as RFA alone for early-stage breast cancer. ${ }^{50,51}$ RFA uses thermal energy to cause coagulative necrosis of tumor. Although there is no specific size cut off, it is more effective for single mass of size less than $2 \mathrm{~cm}^{.52}$ It is contraindicated in invasive lobular carcinoma and intraductal extension of tumor. ${ }^{53}$ Preprocedure ultrasonography, mammography and MRI are required to precisely characterize and localize the mass. Before RFA, sentinel lymph node biopsy should be performed for axillary staging. RFA involves insertion of RFA electrode within the lesion under USG guidance followed by delivery of RFA pulse till completion of ablation seen as completely hyperechoic lesion. Complete ablation and recurrence rate range from 76 to $96.15 \%$ and 1.37 to $14.29 \%$, respectively. ${ }^{54}$ Common complications reported with RFA are skin burns, nipple retraction, muscle burn, and mass formation secondary to fat necrosis. ${ }^{52,55}$ There are technical limitations of RFA which limit its clinical utility such as determining the optimum zone, duration of ablation, accurate assessment of tumor margin if surgery is not performed following RFA. ${ }^{52,54}$ Therefore, treatment outcomes of RFA in terms of long term recurrence and survival rate are still to be evaluate to conclude RFA as an alternative to surgery. The inability to obtain information on the tumor margins following RFA led to discontinuation of the technique in authors' institute.

\section{Accelerated Partial Breast Irradiation}

With increasing rates of early breast cancer detection, breast conservation therapy is often performed which is a combination of BCS with adjuvant whole breast irradiation (WBI). Standard external whole breast irradiation extends over a period of 6 to 6.5 weeks and involves irradiation to the chest wall, lung, and heart in addition to the breast resulting in more complications. ${ }^{56} \mathrm{APBI}$ has emerged as an alternative to WBI-which delivers radiation to the tumor bed at a higher dose per fraction in a shorter time period. It is based on the fact that most cases of tumor recurrence often occur around the tumor margins. Studies have showed that APBI has similar in-breast recurrence rates as WBI. ${ }^{57}$ In ideal setting, APBI is planned with surgery for early nonmetastatic tumors and negative SLNB. Catheters are placed around the surgical margins at the same time before closing the incision. In case the APBI facility is not available in the hospital where surgery is performed, catheters are placed after wound healing (around 3 weeks) under USG guidance around the seroma or scar site with surgical clips serving as markers followed by planning computed tomography (CT) (-Fig. 5).

\section{Interventions with Palliative Intent}

Several complications are seen in patients with metastatic breast cancer that markedly reduce the quality of life. Radiologist can play an important role in the mitigation of suffering caused by these morbid complications

\section{Recurrent Pleural Effusion: Drainage and Pleurodesis}

Breast cancer is the most common cause of malignant pleural effusion in females and often presents with dyspnea. Its management is usually determined by the symptoms, performance status of patient, response to systemic therapy, and lung re-expansion following pleural fluid drainage. Treatment options include observation, therapeutic pleural aspiration, indwelling pleural catheter (IPC), intrapleural instillation of sclerosant, and pleurodesis. Therapeutic thoracentesis is indicated in patients with short survival time, slow fluid re-accumulation, and poor performance status. Indwelling pleural catheter is indicated in patients unsuitable for talc pleurodesis, recurrence after pleurodesis, or trapped lung. ${ }^{58}$
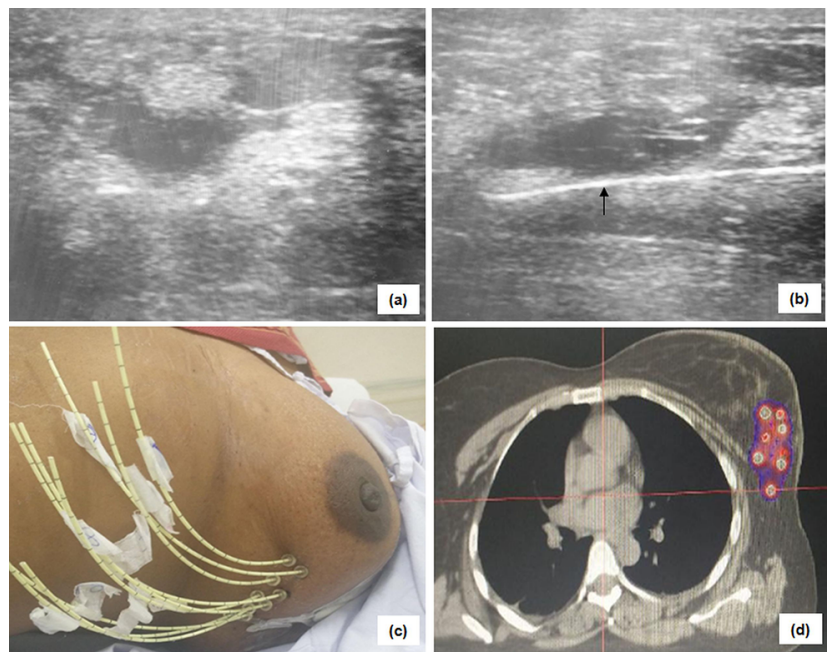

Fig. 5 Accelerated partial breast irradiation (APBI)-postoperative cavity is identified on ultrasound as fluid containing seroma with surrounding architectural distortion (a). Needles have been placed under real time visualization in two planes (b) in which the respective radiation catheters are inserted (c). Planning CT shows these catheters along the postoperative changes and radiation is planned accordingly as shown in figure (d). 
It involves insertion of silicone catheter within the pleural cavity under image guidance (-Fig. 6). It is as effective as pleurodesis in relieving symptoms. IPC-associated pleural infection is the most common complication which is often treated with antibiotic without the removal of catheter. ${ }^{59}$ Pleurodesis involves obliteration of pleural space between visceral and parietal pleura by inflammatory process induced by instillation of mechanical and chemical agents. It is indicated in recurrent malignant pleural effusion in patients with longer life expectancy. Commonly used agents are talc, bleomycin, tetracycline, and doxycycline. ${ }^{60}$ If pleurodesis fails, repeat pleurodesis or IPC can be done.

\section{Pain Management}

Due to the advancement in the management of breast cancer, survival of patient with breast cancer has increased with subsequent rise in morbidity associated with it. One of the most debilitating symptoms related to cancers is pain. Pain can be due to disease (local/metastatic) or secondary to its treatment (surgery/radiotherapy) and is managed mostly conservatively with analgesics and opioids. Chest pain due to pleural effusion is dealt with drainage as described earlier. Perioperative pain can be managed with the addition of regional anesthesia in combination with general anesthesia by means of nerve blocks like thoracic paravertebral, pectoral nerve blocks (PECS) I and II, and serratus anterior (SA) plane blocks. US guidance enables real time visualization of spread of drug, improving the efficacy of local anesthetics. In thoracic paravertebral block, local anesthetic is deposited between pleura and costotransverse ligament approximately 2 to 2.5 - $\mathrm{cm}$ lateral to the tip of spinous process using 18-gauge lumber puncture needle. ${ }^{61}$ It can be given at single level, multiple level, or catheter placement depending on the level or duration of analgesic requirement. Common complication is hypotension due to epidural spread of the drug.

PECS is a recently developed technique of regional anesthesia in which the anesthetic agent is instilled between

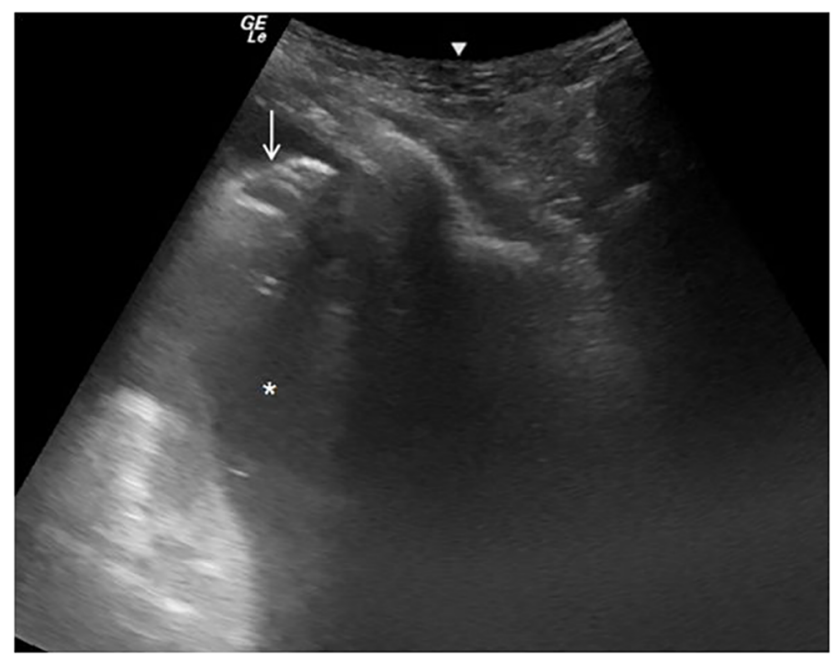

Fig. 6 Pleural effusion drainage: under ultrasound guidance, pigtail (arrow) has been placed within the pleural fluid (asterisk) in a patient with carcinoma breast who presented with sudden onset of breathlessness. pectoralis major and minor to block the median and lateral pectoral nerve. In PECS I, US probe is placed below the lateral third of clavicle in oblique orientation and pectoralis major and minor muscles are identified at the level of third rib. In PECS II, drug is deposited between pectoralis minor and SA at the level of fourth rib to block lateral branch of intercostal nerve, long thoracic nerve, and intercostobrachial nerves. ${ }^{62}$ Serratus anterior plane block is given between the latissimus dorsi and SA muscle in the mid axillary line at the level of fourth/fifth rib to block lateral cutaneous branches of

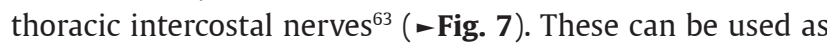
an alternative to thoracic paravertebral block without risk of neuroaxis blockage.

Painful bone metastasis is a common cause of chronic pain in patient with breast cancer. RFA of bone metastasis involves insertion of radiofrequency electrodes under CT guidance and subsequent ablation of tumor under general anesthesia or deep sedation. The procedure usually takes 7 to 12 minutes at an energy level of 90 to $110 \mathrm{~W}$ to achieve the target temperature of 80 to $110^{\circ} \mathrm{C} .{ }^{64}$ It reduces pain by destroying sensory nerve fibers in the periosteum and bone cortex, reduction of lesion volume with decreased stimulation of sensory nerve fibers, and cytokines production. It has been shown that it has resulted in considerable decrease in pain and use of analgesics. ${ }^{65}$ Percutaneous vertebroplasty under fluoroscopic guidance can be performed by injecting bone cement (polymethyl methacrylate) into the vertebral body. It results in significant reduction in pain within 24 hours of pain. ${ }^{66}$ In addition, it increases spine stability and promotes tumor necrosis. ${ }^{67}$

\section{Vision for Future}

\section{VAB to Confirm Complete Pathological Response Post NACT Deferring the Need of Surgery}

With the advancement in NACT regimes, there is significant increase in rates of pathological complete remission. The aim of surgery following NACT is to assess pathological response removing residual disease and achieve locoregional control. Post-chemotherapy changes such as fibrosis, fragmentation, density result in inadequate assessment of residual

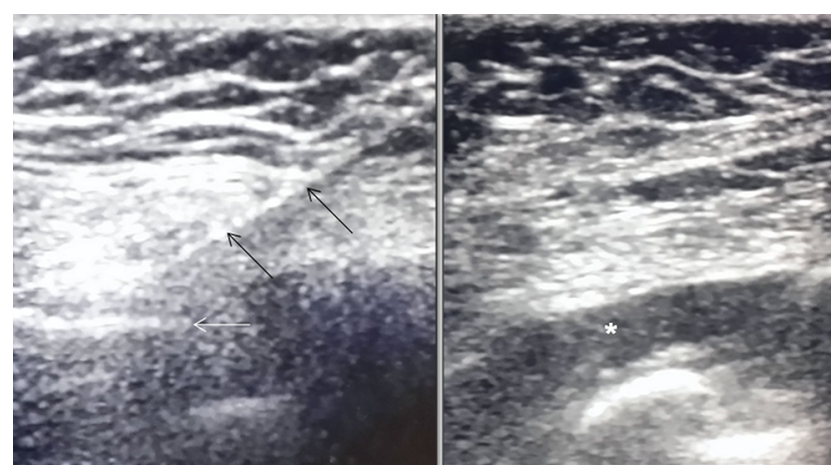

Fig. 7 Serratous anterior block: using real time USG guidance, LP needle (black arrows) is inserted till it reaches the intercostal space or the rib (white arrow). The long-acting local anesthetic agent is injected between the muscles (asterisk) with caution not to injure the pleura. 
tumor. Minimally invasive biopsy methods have been used to assess complete pathological remission. Image-guided VAB has the highest diagnostic accuracy when compared with other methods such as punch biopsy and fine needle biopsy. ${ }^{68,69}$ Combination of imaging and image-guided biopsy has $100 \%$ negative predictive value in diagnosing pathological complete response. ${ }^{68}$ Prospective trials are required to evaluate the need of surgery following pathological complete response diagnosed on VAB.

\section{Post NACT Limited Axillary Dissection}

Management and prognostication of breast cancer depend vastly on the extent of lymph nodes involvement. Since radical axillary lymph node dissection (ALND) is associated with significant morbidity, the concept of sentinel lymph node biopsy came into being which aimed at removing only the nodes showing uptake of dye. Recent studies have shown USG as equally accurate in detecting abnormal nodes and have suggested only removal of these nodes, especially in post NACT patients..$^{70-75}$ Lymph nodes are categorized as suspicious if they show: loss of fatty hilum/asymmetrical cortical thickening/abnormal cortex to hilum ratio/peripheral blood flow, on USG. The most suspicious lymph node is then subjected to guided biopsy and placement of marker clip. If malignancy is proven histopathologically within these nodes, the clip serves as target for localization and dedicated removal of the involved nodes can be performed instead of subjecting the patient to morbid ALND. ${ }^{76}$

To summarize, breast imaging has emerged as a special branch of radiology where radiologist plays a crucial role not only in diagnosis but also in treatment and follow-up of the patient.

\section{Financial Support \\ None.}

\section{Conflict of Interest}

None declared.

\section{References}

1 GLOBACON 2020. Global Health Observatory. Geneva: World Health Organization. Available at: https://gco.iarc.fr/today/ data/factsheets/populations/356-india-fact-sheets.pdf. Accessed July 23, 2021

2 Bray F, McCarron P, Parkin DM. The changing global patterns of female breast cancer incidence and mortality. Breast Cancer Res 2004;6(6):229-239

3 Hari S, Kumari S, Srivastava A, Thulkar S, Mathur S, Veedu PT. Image guided versus palpation guided core needle biopsy of palpable breast masses: a prospective study. Indian J Med Res 2016;143(5):597-604

4 Teshome M, Hunt KK. Neoadjuvant therapy in the treatment of breast cancer. Surg Oncol Clin N Am 2014;23(3):505-523

5 Collaço LM, de Lima RS, Werner B, Torres LF. Value of fine needle aspiration in the diagnosis of breast lesions. Acta Cytol 1999;43(4):587-592

6 Parker SH, Jobe WE, Dennis MA, et al. US-guided automated large-core breast biopsy. Radiology 1993;187(2):507-511

7 Burbank F, Parker SH, Fogarty TJ. Stereotactic breast biopsy: improved tissue harvesting with the Mammotome. Am Surg 1996;62(9):738-744
8 Jackman RJ, Marzoni FA Jr, Rosenberg J. False-negative diagnoses at stereotactic vacuum-assisted needle breast biopsy: long-term follow-up of 1,280 lesions and review of the literature. AJR Am J Roentgenol 2009;192(2):341-351

9 Zhang C, Lewis DR, Nasute P, Hayes M, Warren LJ, Gordon PB. The negative predictive value of ultrasound-guided 14-gauge core needle biopsy of breast masses: a validation study of 339 cases. Cancer Imaging 2012;12:488-496

10 Lee SH, Kim E-K, Kim MJ, Moon HJ, Yoon JH. Vacuum-assisted breast biopsy under ultrasonographic guidance: analysis of a 10-year experience. Ultrasonography 2014;33(4):259-266

11 Huang ML, Adrada BE, Candelaria R, Thames D, Dawson D, Yang WT. Stereotactic breast biopsy: pitfalls and pearls. Tech Vasc Interv Radiol 2014;17(1):32-39

12 Schrading S, Distelmaier M, Dirrichs T, et al. Digital breast tomosynthesis-guided vacuum-assisted breast biopsy: initial experiences and comparison with prone stereotactic vacuum-assisted biopsy. Radiology 2015;274(3):654-662

13 Rochat CJ, Baird GL, Lourenco AP. Digital mammography stereotactic biopsy versus digital breast tomosynthesis-guided biopsy: differences in biopsy targets, pathologic results, and discordance rates. Radiology 2020;294(3):518-527

14 Bahl M, Maunglay M, D’Alessandro HA, Lehman CD. Comparison of upright digital breast tomosynthesis-guided versus prone stereotactic vacuum-assisted breast biopsy. Radiology 2019;290(2):298-304

15 Carrara GF, Scapulatempo-Neto C, Abrahão-Machado LF, et al. Breast-conserving surgery in locally advanced breast cancer submitted to neoadjuvant chemotherapy. Safety and effectiveness based on ipsilateral breast tumor recurrence and long-term follow-up. Clinics (São Paulo 2017;72(3):134-142

16 Baron LF, Baron PL, Ackerman SJ, Durden DD, Pope TL Jr. Sonographically guided clip placement facilitates localization of breast cancer after neoadjuvant chemotherapy. AJR Am J Roentgenol 2000;174(2):539-540

17 Pennisi A, Kieber-Emmons T, Makhoul I, Hutchins L. Relevance of pathological complete response after neoadjuvant therapy for breast cancer. Breast Cancer (Auckl 2016;10:103-106

18 Margolin FR, Kaufman L, Denny SR, Jacobs RP, Schrumpf JD. Metallic marker placement after stereotactic core biopsy of breast calcifications: comparison of two clips and deployment techniques. AJR Am J Roentgenol 2003;181(6):1685-1690

19 Espinosa-Bravo M, Sao Avilés A, Esgueva A, et al. Breast conservative surgery after neoadjuvant chemotherapy in breast cancer patients: comparison of two tumor localization methods. Eur J Surg Oncol 2011;37(12):1038-1043

20 Alonso-Bartolome P, Ortega Garcia E, Garijo Ayensa F, de Juan Ferre A, Vega Bolivar A. Utility of the tumor bed marker in patients with breast cancer receiving induction chemotherapy. Acta Radiol 2002;43(1):29-33

21 Braeuning MP, Burke ET, Pisano ED. Embolization coils as tumor markers for mammography in patients undergoing neoadjuvant chemotherapy for carcinoma of the breast. AJR Am J Roentgenol 2000;174(1):251-252

22 Zhang Y, Seely J, Cordeiro E, et al. Radioactive seed localization versus wire-guided localization for nonpalpable breast cancer: a cost and operating room efficiency analysis. Ann Surg Oncol 2017;24(12):3567-3573

23 Hayes MK. Update on preoperative breast localization. Radiol Clin North Am 2017;55(3):591-603

24 Oh JL, Nguyen G, Whitman GJ, et al. Placement of radiopaque clips for tumor localization in patients undergoing neoadjuvant chemotherapy and breast conservation therapy. Cancer 2007;110(11):2420-2427

25 Kirstein LJ, Rafferty E, Specht MC, et al. Outcomes of multiple wire localization for larger breast cancers: when can mastectomy be avoided? J Am Coll Surg 2008;207(3):342-346 
26 Liberman L, Kaplan J, Van Zee KJ, et al. Bracketing wires for preoperative breast needle localization. AJR Am J Roentgenol 2001;177(3):565-572

27 Demiral G, Senol M, Bayraktar B, Ozturk H, Celik Y, Boluk S. Diagnostic value of hook wire localization technique for non-palpable breast lesions. J Clin Med Res 2016;8(5):389-395

28 Bronstein AD, Kilcoyne RF, Moe RE. Complications of needle localization of foreign bodies and nonpalpable breast lesions. Arch Surg 1988;123(6):775-779

29 Corsi F, Sorrentino L, Bossi D, Sartani A, Foschi D. Preoperative localization and surgical margins in conservative breast surgery. Int J Surg Oncol 2013;2013:793819

30 Hughes JH, Mason MC, Gray RJ, et al. A multi-site validation trial of radioactive seed localization as an alternative to wire localization. Breast J 2008;14(2):153-157

31 Pavlicek W, Walton HA, Karstaedt PJ, Gray RJ. Radiation safety with use of I-125 seeds for localization of nonpalpable breast lesions. Acad Radiol 2006;13(7):909-915

32 Sharek D, Zuley ML, Zhang JY, Soran A, Ahrendt GM, Ganott MA. Radioactive seed localization versus wire localization for lumpectomies: a comparison of outcomes. AJR Am J Roentgenol 2015;204(4):872-877

33 Harvey JR, Lim Y, Murphy J, et al. Safety and feasibility of breast lesion localization using magnetic seeds (Magseed): a multi-centre, open-label cohort study. Breast Cancer Res Treat 2018;169(3):531-536

34 Rahusen FD, Bremers AJ, Fabry HF. van Amerongen AH, Boom RP, Meijer S. Ultrasound-guided lumpectomy of nonpalpable breast cancer versus wire-guided resection: a randomized clinical trial. Ann Surg Oncol 2002;9(10):994-998

35 Hu X, Li S, Jiang Y, et al. Intraoperative ultrasound-guided lumpectomy versus wire-guided excision for nonpalpable breast cancer. J Int Med Res 2020;48(1):300060519896707

36 Krekel NM, Haloua MH, Lopes Cardozo AM, et al. Intraoperative ultrasound guidance for palpable breast cancer excision (COBALT trial): a multicentre, randomised controlled trial. Lancet Oncol 2013;14(1):48-54

37 Vispute T, Seenu V, Parshad R, Hari S, Thulkar S, Mathur S; Suhani. Comparison of resection margins and cosmetic outcome following intraoperative ultrasound-guided excision versus conventional palpation-guided breast conservation surgery in breast cancer: a randomized controlled trial. Indian J Cancer 2018;55(4):361-365

38 Rinaldi P, Ierardi C, Costantini M, et al. Cystic breast lesions: sonographic findings and clinical management. J Ultrasound Med 2010;29(11):1617-1626

39 Gomes C, Amaral N, Marques C. Borralho R, de Oliveira CF. Sclerosis of gross cysts of the breast: a three-year study. Eur J Gynaecol Oncol 2002;23(3):191-194

40 Gizienski T-A, Harvey JA, Sobel AH. Breast cyst recurrence after postaspiration injection of air. Breast J 2002;8(1):34-37

41 Özgen A. Effectiveness of single-session ultrasound-guided percutaneous ethanol sclerotherapy in simple breast cysts. Diagn Interv Radiol 2016;22(3):220-223

42 Imperiale A, Zandrino F, Calabrese M, Parodi G, Massa T. Abscesses of the breast. US-guided serial percutaneous aspiration and local antibiotic therapy after unsuccessful systemic antibiotic therapy. Acta Radiol 2001;42(2):161-165

43 Ulitzsch D, Nyman MKG, Carlson RA. Breast abscess in lactating women: US-guided treatment. Radiology 2004;232(3):904-909

44 Trop I, Dugas A, David J, et al. Breast abscesses: evidence-based algorithms for diagnosis, management, and follow-up. Radiographics 2011;31(6):1683-1699

45 Hahn M, Krainick-Strobel U, Toellner T, et al; Minimally Invasive Breast Intervention Study Group (AG MiMi) of the German Society of Senology (DGS)Study Group for Breast Ultrasonography of the German Society for Ultrasound in
Medicine (DEGUM). Interdisciplinary consensus recommendations for the use of vacuum-assisted breast biopsy under sonographic guidance: first update 2012. Ultraschall Med 2012;33(4):366-371

46 Yom CK, Moon BI, Choe KJ, Choi HY, Park YL. Long-term results after excision of breast mass using a vacuum-assisted biopsy device. ANZ J Surg 2009;79(11):794-798

47 Wang ZL, Li JL, Su L, Zhang YF, Tang J. An evaluation of a 10-gauge vacuum-assisted system for ultrasound-guided excision of clinically benign breast lesions. Breast 2009;18(3):192-196

48 Krainick-Strobel U, Huber B, Majer I, et al. Complete extirpation of benign breast lesions with an ultrasound-guided vacuum biopsy system. Ultrasound Obstet Gynecol 2007;29(3):342-346

49 Luo HJ, Chen X, Tu G, Wang J, Wu CY, Yang GL. Therapeutic application of ultrasound-guided 8-gauge Mammotome system in presumed benign breast lesions. Breast J 2011;17(5):490-497

50 Burak WE Jr, Agnese DM, Povoski SP, et al. Radiofrequency ablation of invasive breast carcinoma followed by delayed surgical excision. Cancer 2003;98(7):1369-1376

51 Kinoshita T, Iwamoto E, Tsuda H, Seki K. Radiofrequency ablation as local therapy for early breast carcinomas. Breast Cancer 2011;18(1):10-17

52 Ito $\mathrm{T}$, Oura $\mathrm{S}$, Nagamine $\mathrm{S}$, et al. Radiofrequency ablation of breast cancer: a retrospective study. Clin Breast Cancer 2018;18(4):e495-e500

53 Palussière J, Henriques C, Mauriac L, et al. Radiofrequency ablation as a substitute for surgery in elderly patients with nonresected breast cancer: pilot study with long-term outcomes. Radiology 2012;264(2):597-605

54 Chen J, Zhang C, Li F, et al. A meta-analysis of clinical trials assessing the effect of radiofrequency ablation for breast cancer. OncoTargets Ther 2016;9:1759-1766

55 Nguyen T, Hattery E, Khatri VP. Radiofrequency ablation and breast cancer: a review. Gland Surg 2014;3(2):128-135

56 Hoover S, Bloom E, Patel S. Review of breast conservation therapy: then and now. ISRN Oncol 2011;2011:617593

57 Vicini F, Beitsch P, Quiet C, et al. Five-year analysis of treatment efficacy and cosmesis by the American Society of Breast Surgeons MammoSite Breast Brachytherapy Registry Trial in patients treated with accelerated partial breast irradiation. Int J Radiat Oncol Biol Phys 2011;79(3):808-817

58 Roberts ME, Neville E, Berrisford RG, Antunes G, Ali NJ; BTS Pleural Disease Guideline Group. Management of a malignant pleural effusion: British Thoracic Society pleural disease guideline 2010. Thorax 2010;65(suppl 2) :ii32-ii40

59 Fysh ETH, Tremblay A, Feller-Kopman D, et al. Clinical outcomes of indwelling pleural catheter-related pleural infections: an international multicenter study. Chest 2013;144(5):1597-1602

60 Bhatnagar R, Laskawiec-Szkonter M, Piotrowska HEG, et al. Evaluating the efficacy of thoracoscopy and talc poudrage versus pleurodesis using talc slurry (TAPPS trial): protocol of an open-label randomised controlled trial. BMJ Open 2014;4(11):e007045

61 O Riain SC, Donnell BO, Cuffe T, Harmon DC, Fraher JP, Shorten G. Thoracic paravertebral block using real-time ultrasound guidance. Anesth Analg 2010;110(1):248-251

62 Bashandy GMN, Abbas DN. Pectoral nerves I and II blocks in multimodal analgesia for breast cancer surgery: a randomized clinical trial. Reg Anesth Pain Med 2015;40(1):68-74

63 Blanco R, Parras T, McDonnell JG, Prats-Galino A. Serratus plane block: a novel ultrasound-guided thoracic wall nerve block. Anaesthesia 2013;68(11):1107-1113

64 Botsa E, Mylona S, Koutsogiannis I, Koundouraki A, Thanos L. CT image guided thermal ablation techniques for palliation of painful bone metastases. Ann Palliat Med 2014;3(2):47-53

65 Goetz MP, Callstrom MR, Charboneau JW, et al. Percutaneous image-guided radiofrequency ablation of painful 
metastases involving bone: a multicenter study. J Clin Oncol 2004;22(2):300-306

66 Gangi A, Sabharwal T, Irani FG, Buy X, Morales JP, Adam A; Standards of Practice Committee of the Society of Interventional Radiology. Quality assurance guidelines for percutaneous vertebroplasty. Cardiovasc Intervent Radiol 2006;29(2):173-178

67 Yang H-L, Sun Z-Y, Wu G-Z, Chen K-W, Gu Y, Qian Z-L. Do vertebroplasty and kyphoplasty have an antitumoral effect? Med Hypotheses 2011;76(1):145-146

68 Kuerer HM, Rauch GM, Krishnamurthy S, et al. A clinical feasibility trial for identification of exceptional responders in whom breast cancer surgery can be eliminated following neoadjuvant systemic therapy. Ann Surg 2018;267(5):946-951

69 Heil J, Kümmel S, Schaefgen B, et al. Diagnosis of pathological complete response to neoadjuvant chemotherapy in breast cancer by minimal invasive biopsy techniques. $\mathrm{Br}$ J Cancer 2015;113(11):1565-1570

70 Giuliano AE, Hunt KK, Ballman KV, et al. Axillary dissection vs no axillary dissection in women with invasive breast cancer and sentinel node metastasis: a randomized clinical trial. JAMA 2011;305(6):569-575

71 Henke G, Knauer M, Ribi K, et al. Tailored axillary surgery with or without axillary lymph node dissection followed by radiotherapy in patients with clinically node-positive breast cancer (TAXIS): study protocol for a multicenter, randomized phase-III trial. Trials 2018;19(1):667

72 Carter S, Neuman H, Mamounas EP, et al. Debating the optimal approach to nodal management after pathologic complete response to neoadjuvant chemotherapy in patients with breast cancer. Am Soc Clin Oncol Educ Book 2019;39:42-48

73 Lee J, Choi JE, Kim SJ, et al. Korean Breast Cancer Society. Comparative study between sentinel lymph node biopsy and axillary dissection in patients with one or two lymph node metastases. J Breast Cancer 2018;21(3):306-314

74 Verheuvel NC, Voogd AC, Tjan-Heijnen VCG, Roumen RMH. Potential impact of application of Z0011 derived criteria to omit axillary lymph node dissection in node positive breast cancer patients. Eur J Surg Oncol 2016;42(8):1162-1168

75 Haffty BG, McCall LM, Ballman KV, Buchholz TA, Hunt KK, Boughey JC. Impact of radiation on locoregional control in women with node-positive breast cancer treated with neoadjuvant chemotherapy and axillary lymph node dissection: results from ACOSOG Z1071 clinical trial. Int J Radiat Oncol Biol Phys 2019;105(1):174-182

76 Shin K, Caudle AS, Kuerer HM, et al. Radiologic mapping for targeted axillary dissection: needle biopsy to excision. AJR Am J Roentgenol 2016;207(6):1372-1379 